PROCEEDINGS OF THE

AMERICAN MATHEMATICAL SOCIETY

Volume 133, Number 6, Pages 1777-1785

S 0002-9939(04)07733-0

Article electronically published on December 20, 2004

\title{
BOUNDEDNESS OF ADMISSIBLE AREA FUNCTION ON NONISOTROPIC LIPSCHITZ SPACE
}

\author{
JINSHOU GAO AND HOUYU JIA \\ (Communicated by Joseph A. Ball)
}

\begin{abstract}
Let $B$ be the unit ball in $C^{n}$, let $S$ be the unit sphere, and let $S_{\beta}(f)$ be the admissible area function. In this paper, we show that if $f \in \operatorname{Lip}_{\alpha}(S)$, then $S_{\beta}(f) \in \operatorname{Lip}_{\alpha}(S)$ and there exists a constant $C$ such that $\left\|S_{\beta}(f)\right\|_{L i p_{\alpha}} \leq C\|f\|_{L i p_{\alpha}}$.
\end{abstract}

\section{INTRODUCTION}

Let $B$ be the unit ball in $C^{n}, d \nu$ the normalized Lebesgue measure on $B, d \sigma$ the normalized surface measure on the boundary $S$ of $B$.

For $\xi \in S$ and $0<\delta \leq 2$, let $Q_{\delta}(\xi)=\{\eta \in S: d(\xi, \eta)=|1-\langle\xi, \eta\rangle|<\delta\}$ be a nonisotropic ball of $S$.

We denote by $f \in \operatorname{Lip}_{\alpha}(S)$ the nonisotropic Lipschitz space of order $0<\alpha<1$ if

$$
\sup _{\xi, \eta \in S} \frac{|f(\xi)-f(\eta)|}{d(\xi, \eta)^{\alpha}}:=\|f\|_{L i p_{\alpha}}<\infty .
$$

We shall follow the convention of identifying the function $f$ on the unit sphere with invariant harmonic extensions into the unit ball defined via the Poisson formula:

$$
f(z)=\int_{S} P(z, \xi) f(\xi) d \sigma(\xi)
$$

where $P(z, \xi)$ is the Poisson-Szegö kernel

$$
P(z, \xi)=\frac{\left(1-|z|^{2}\right)^{n}}{|1-\langle z, \xi\rangle|^{2 n}} .
$$

For $F \in C^{1}(B)$, let $D F=\left(\frac{\partial F}{\partial x_{1}}, \frac{\partial F}{\partial y_{1}}, \cdots, \frac{\partial F}{\partial x_{n}}, \frac{\partial F}{\partial y_{n}}\right)(k=1,2, \cdots, n)$ be the real gradient of $F$ and $\nabla F=\left(\frac{\partial F}{\partial z_{1}}, \frac{\partial F}{\partial z_{2}}, \cdots, \frac{\partial F}{\partial z_{n}}\right)$ the complex gradient of $F, z_{k}=$ $x_{k}+i y_{k}$.

Let $\widetilde{\nabla}$ denote the invariant gradient on $B$, that is,

$$
\widetilde{\nabla} f(z)=\nabla\left(f \circ \varphi_{z}\right)(0),
$$

Received by the editors September 17, 2003 and, in revised form, February 20, 2004.

2000 Mathematics Subject Classification. Primary 47B38, 32A37, 42B25.

Key words and phrases. Unit ball in $C^{n}$, admissible area function, nonisotropic Lipschitz space.

The second author was supported in part by the Education Department of Zhejiang Province.

(C)2004 American Mathematical Society

Reverts to public domain 28 years from publication 
where $\varphi_{z}$ is the involution automorphism of $B$ satisfying $\varphi_{z}(0)=z, \varphi_{z}(z)=0$. It has been shown in [1] that for $a \in B$,

$$
\varphi_{a}(z)=\frac{a-P_{a} z-\left(1-|a|^{2}\right)^{\frac{1}{2}} Q_{a} z}{1-\langle z, a\rangle}
$$

where $P_{a} z=\frac{\langle z, a\rangle}{|a|^{2}} a, P_{0} z=0, Q_{a}=I-P_{a}$ and

$$
\varphi_{a}^{\prime}(0)=-\left(1-|a|^{2}\right) P_{a}-\sqrt{1-|a|^{2}} Q_{a} .
$$

Now, by a simple computation we get that

$$
\begin{aligned}
|\widetilde{\nabla} f(z)|^{2} & =\left|\nabla\left(f \circ \varphi_{z}\right)(0)\right|^{2}=\left|\nabla f(z) \varphi_{z}^{\prime}(0)\right|^{2}=\left|\varphi_{\bar{z}}^{\prime}(0) \nabla f(z)\right|^{2} \\
& =\left(1-|z|^{2}\right)^{2}\left|P_{\bar{z}} \nabla f(z)\right|^{2}+\left(1-|z|^{2}\right)\left|Q_{\bar{z}} \nabla f(z)\right|^{2} \\
& =\left(1-|z|^{2}\right)\left(|\nabla f(z)|^{2}-|\langle\nabla f(z), \bar{z}\rangle|^{2}\right) \\
& =\left(1-|z|^{2}\right)\left(|\nabla f(z)|^{2}-|R f(z)|^{2}\right)
\end{aligned}
$$

where $R f$ is the radial derivative of $f$.

If $u \in C^{1}(B)$, the admissible area function is defined on $S$ by

$$
S_{\beta} u(\xi)=\left\{\int_{D_{\beta}(\xi)}|\widetilde{\nabla} u|^{2}\left(1-|z|^{2}\right)^{-(n+1)} d \nu(z)\right\}^{\frac{1}{2}}
$$

where $D_{\beta}(\xi)$ denotes the admissible approach region with $\beta>1$ :

$$
D_{\beta}(\xi)=\left\{z \in B:|1-\langle z, \xi\rangle|<\frac{\beta}{2}\left(1-|z|^{2}\right)\right\} .
$$

In [2] S. Y. Chang proved the boundedness of $S_{\beta}$ on $L^{p}(1<p<+\infty)$; P. Ahern and J. Bruna [3] studied the characterization of Hardy-Sobolev spaces by $S_{\beta}(f)$.

The aim of this paper is to study the behavior of $S_{\beta}(f)$ where $f \in \operatorname{Lip}_{\alpha}(S)(0<$ $\alpha<1)$.

Theorem 1.1. If $f \in \operatorname{Lip}_{\alpha}(S)(0<\alpha<1)$ and $S_{\beta}(f)<+\infty$ a.e.on $S$, then $S_{\beta} f \in \operatorname{Lip}_{\alpha}(S)$ and there exists a constant $C$ such that

$$
\left\|S_{\beta}(f)\right\|_{L i p_{\alpha}} \leq C\|f\|_{L i p_{\alpha}} .
$$

Throughout this paper we shall use the letter $C$ to denote constants, and it may change from line to line.

\section{Preliminaries}

Lemma 2.1. Let $z \in B, \xi \in S$. We have

$$
|\widetilde{\nabla} P(z, \xi)| \leq n\left(\frac{\left(1-|z|^{2}\right)^{n}}{|1-\langle z, \xi\rangle|^{2 n}}+\frac{\left(1-|z|^{2}\right)^{n+1}}{|1-\langle z, \xi\rangle|^{2 n+1}}+\frac{\left(1-|z|^{2}\right)^{n+\frac{1}{2}}}{|1-\langle z, \xi\rangle|^{2 n+\frac{1}{2}}}\right) .
$$

Proof. Notice firstly that $P(z, \xi)=\bar{P}(z, \xi)$ and

$$
|\widetilde{D} P(z, \xi)|=|\widetilde{\nabla} P(z, \xi)| .
$$

To calculate $|\widetilde{\nabla} P(z, \xi)|$ we will use formula (1.1). We have

$$
\nabla P(z, \xi)=-n\left(\frac{\left(1-|z|^{2}\right)^{n-1}}{|1-\langle z, \xi\rangle|^{2 n}} \bar{z}-\frac{\left(1-|z|^{2}\right)^{n}(1-\overline{\langle z, \xi\rangle})}{|1-\langle z, \xi\rangle|^{2 n+2}} \bar{\xi}\right)
$$


and

$$
\begin{aligned}
P_{\bar{z}} \nabla P(z, \xi) & =-n\left(\frac{\left(1-|z|^{2}\right)^{n-1}}{|1-\langle z, \xi\rangle|^{2 n}}-\frac{\left(1-|z|^{2}\right)^{n}(1-\overline{\langle z, \xi\rangle})\langle\bar{\xi}, \bar{z}\rangle}{|z|^{2}|1-\langle z, \xi\rangle|^{2 n+2}}\right) \bar{z} \\
Q_{\bar{z}} \nabla P(z, \xi) & =-n \frac{\left(1-|z|^{2}\right)^{n}(1-\overline{\langle z, \xi\rangle})\left(|z|^{2} \bar{\xi}-\langle\bar{\xi}, \bar{z}\rangle \bar{z}\right)}{|z|^{2}|1-\langle z, \xi\rangle|^{2 n+2}} .
\end{aligned}
$$

Hence

$$
\begin{aligned}
|\widetilde{\nabla} P(z, \xi)| & \leq\left(1-|z|^{2}\right)\left|P_{\bar{z}} \nabla P(z, \xi)\right|+\left(1-|z|^{2}\right)^{\frac{1}{2}}\left|Q_{\bar{z}} \nabla P(z, \xi)\right| \\
& \leq n\left(\frac{\left(1-|z|^{2}\right)^{n}}{|1-\langle z, \xi\rangle|^{2 n}}+\frac{\left(1-|z|^{2}\right)^{n+1}}{|1-\langle z, \xi\rangle|^{2 n+1}}+\frac{\left(1-|z|^{2}\right)^{n+\frac{1}{2}}}{|1-\langle z, \xi\rangle|^{2 n+\frac{1}{2}}}\right) .
\end{aligned}
$$

The following lemma is well known; see [4].

Lemma 2.2. Let $f \in L^{1}(S)$ and $0<\alpha<1$. Then the norm $\|f\|_{L i p_{\alpha}}$ is equivalent to

$$
\sup _{Q} \frac{1}{|Q|^{1+\frac{\alpha}{n}}} \int_{Q}\left|f-f_{Q}\right| d \sigma
$$

where $f_{Q}=\frac{1}{|Q|} \int_{Q} f(\xi) d \sigma(\xi)$.

Lemma 2.3. Let $f \in \operatorname{Lip}_{\alpha}(S), \gamma \geq 0$. Let $Q_{0}$ be a nonisotropic ball of radius $\delta>0$ and center $\eta^{*}$. If $\left|1-\left\langle\eta, \eta^{*}\right\rangle\right|<\frac{\delta}{16}$, then there exists a constant $C$ depending only on $n, \gamma$ so that for any $z \in B$ that satisfies $|1-\langle z, \eta\rangle|<\frac{\delta}{16}$, we have

$$
\int_{S \backslash Q_{0}} \frac{\left|f(\xi)-f_{Q_{0}}\right|}{|1-\langle z, \xi\rangle|^{2 n+\gamma}} d \sigma(\xi) \leq C \delta^{-(n+\gamma-\alpha)}\|f\|_{L i p_{\alpha}} .
$$

Proof. For $\xi \in S \backslash Q_{0}$ and $z \in B$ that satisfies $|1-\langle z, \eta\rangle|<\frac{\delta}{16},\left|1-\left\langle\eta, \eta^{*}\right\rangle\right|<\frac{\delta}{16}$, we have

$$
\begin{aligned}
\left|f(\xi)-f_{Q_{0}}\right| & \leq \frac{1}{\left|Q_{0}\right|} \int_{Q_{0}}\left|f(\xi)-f\left(\eta^{\prime}\right)\right| d \sigma\left(\eta^{\prime}\right) \\
& \leq \frac{1}{\left|Q_{0}\right|} \int_{Q_{0}}\left|1-\left\langle\xi, \eta^{\prime}\right\rangle\right|^{\alpha} d \sigma\left(\eta^{\prime}\right)\|f\|_{L i p_{\alpha}} \\
& \leq C|1-\langle z, \xi\rangle|^{\alpha}\|f\|_{L i p_{\alpha}} .
\end{aligned}
$$

Hence

$$
\begin{aligned}
& \int_{S \backslash Q_{0}} \frac{\left|f(\xi)-f_{Q_{0}}\right|}{|1-\langle z, \xi\rangle|^{2 n+\gamma}} d \sigma(\xi) \\
& \leq C\|f\|_{L i p_{\alpha}} \int_{S \backslash Q_{0}} \frac{1}{|1-\langle z, \xi\rangle|^{2 n+\gamma-\alpha}} d \sigma(\xi) \\
& \leq C \delta^{-(n+\gamma-\alpha)}\|f\|_{L i p_{\alpha}} .
\end{aligned}
$$

To be convenient, we denote $r(z)=\left(1-|z|^{2}\right)(z \in B)$. 
Lemma 2.4. Let $Q_{0}$ be a nonisotropic ball of radius $\delta>0$ and center $\eta^{*}$, let $R$ be a nonisotropic ball of radius $r(z)$ and center at $\eta^{*}$, and let $f \in \operatorname{Lip} \alpha(S)$. Then

$$
\begin{array}{rlrl}
\left|f_{R}-f_{Q_{0}}\right| & \leq C\left(1+\left|\ln \left[\frac{r(z)}{\delta}\right]\right|\right) r(z)^{\alpha}\|f\|_{L i p_{\alpha}} & (r(z)>\delta), \\
\left|f_{R}-f_{Q_{0}}\right| \leq C\left(1+\left|\ln \left[\frac{r(z)}{\delta}\right]\right|\right) \delta^{\alpha}\|f\|_{L i p_{\alpha}} & (r(z)<\delta) .
\end{array}
$$

Proof. We only prove (2.1), the proof of (2.2) being similar.

Since $r(z)>\delta$, we choose $k$ such that $2^{k} \delta<r(z) \leq 2^{k+1} \delta$. Then $\frac{2^{k+1} \delta}{r(z)} \leq 2$ and $k \leq \log _{2} \frac{r(z)}{\delta}$, and

$$
\begin{aligned}
\left|f_{R}-f_{Q_{0}}\right| & =\left|\frac{1}{|R|} \int_{R}\left(f(\xi)-f_{Q_{0}}\right) d \sigma(\xi)\right| \\
& \leq C(r(z))^{-n} \int_{R}\left|f(\xi)-f_{Q_{0}}\right| d \sigma(\xi) \\
& \leq C(r(z))^{-n} \int_{Q_{k+1}}\left|f(\xi)-f_{Q_{0}}\right| d \sigma(\xi) .
\end{aligned}
$$

As in the proof of Lemma 2.3 we have

$$
\begin{aligned}
\int_{Q_{k+1}}\left|f(\xi)-f_{Q_{0}}\right| d \sigma(\xi) & \leq C(k+2)\left|Q_{k+1}\right|^{1+\frac{\alpha}{n}}\|f\|_{L i p_{\alpha}} \\
& \leq C(k+2)\left|2^{k+1} \delta\right|^{n+\alpha}\|f\|_{L i p_{\alpha}} .
\end{aligned}
$$

Thus

$$
\begin{aligned}
\left|f_{R}-f_{Q_{0}}\right| & \leq C(k+2)\left|2^{k+1} \delta\right|^{n+\alpha}(r(z))^{-n}\|f\|_{L i p_{\alpha}} \\
& \leq C 2^{n+\alpha}\left(2+\left|\log _{2}\left[\frac{r(z)}{\delta}\right]\right|\right)(r(z))^{\alpha}\|f\|_{L i p_{\alpha}} .
\end{aligned}
$$

Since $1+\left|\ln \left[\frac{r(z)}{\delta}\right]\right|$ is equivalent to $2+\left|\log _{2}\left[\frac{r(z)}{\delta}\right]\right|$, 2.1) is proved, which completes the proof of the lemma.

Lemma 2.5. Let $R^{\prime}$ be a nonisotropic ball of radius $4 \alpha r(z)$ and center at $\eta^{*}$, and let $f \in \operatorname{Lip}_{\alpha}(S)$. Then

$$
\int_{S} \frac{\left|f(\xi)-f_{R^{\prime}}\right|}{|1-\langle z, \xi\rangle|^{2 n+\gamma}} d \sigma(\xi) \leq C(r(z))^{-(n+\gamma-\alpha)}\|f\|_{L i p_{\alpha}} .
$$

Proof. Argue as in the proof of Lemma 2.3.

Lemma 2.6. Let $Q_{0}$ be a nonisotropic ball of radius $\delta$ and center at $\eta^{*}$. If $\frac{\beta}{2} r(z) \geq$ $\frac{\delta}{16},\left|1-\left\langle\eta, \eta^{*}\right\rangle\right|<\frac{\delta}{16},|1-\langle z, \eta\rangle|<\frac{\beta}{2} r(z)$, we have

$$
\int_{S} \frac{\left|f(\xi)-f_{Q_{0}}\right|}{|1-\langle z, \xi\rangle|^{2 n+\gamma}} d \sigma(\xi) \leq C(r(z))^{-(n+\gamma-\alpha)}\left(1+\left|\ln \left[\frac{r(z)}{\delta}\right]\right|\right)\|f\|_{L i p_{\alpha}} .
$$

Proof.

$$
\int_{S} \frac{\left|f(\xi)-f_{Q_{0}}\right|}{|1-\langle z, \xi\rangle|^{2 n+\gamma}} d \sigma(\xi) \leq \int_{S} \frac{\left|f(\xi)-f_{R^{\prime}}\right|}{|1-\langle z, \xi\rangle|^{2 n+\gamma}} d \sigma(\xi)+\left|f_{R^{\prime}}-f_{Q_{0}}\right| \int_{S} \frac{d \sigma(\xi)}{|1-\langle z, \xi\rangle|^{2 n+\gamma}} .
$$


By Lemma 2.4 and Lemma 2.5, we have

$$
\begin{aligned}
& \int_{S} \frac{\left|f(\xi)-f_{R^{\prime}}\right|}{|1-\langle z, \xi\rangle|^{2 n+\gamma}} d \sigma(\xi) \leq C(r(z))^{-(n+\gamma-\alpha)}\|f\|_{L i p_{\alpha}}, \\
& \left|f_{R^{\prime}}-f_{Q_{0}}\right| \leq C(r(z))^{\alpha}\left(1+\left|\ln \left[\frac{r(z)}{\delta}\right]\right|\right)\|f\|_{L i p_{\alpha}} .
\end{aligned}
$$

We also have (see [1] P17])

$$
\int_{S} \frac{d \sigma(\xi)}{|1-\langle z, \xi\rangle|^{2 n+\gamma}} \approx r(z)^{-(n+\gamma)}
$$

Hence

$$
\begin{aligned}
& \int_{S} \frac{\left|f(\xi)-f_{Q_{0}}\right|}{|1-\langle z, \xi\rangle|^{2 n+\gamma}} d \sigma(\xi) \\
& \leq C(r(z))^{-(n+\gamma-\alpha)}\|f\|_{L i p_{\alpha}}+C(r(z))^{-(n+\gamma-\alpha)}\left(1+\left|\ln \left[\frac{r(z)}{\delta}\right]\right|\right)\|f\|_{L i p_{\alpha}} \\
& \leq C(r(z))^{-(n+\gamma-\alpha)}\left(1+\left|\ln \left[\frac{r(z)}{\delta}\right]\right|\right)\|f\|_{L_{i p_{\alpha}} .}
\end{aligned}
$$

\section{The Proof of Theorem 1.1}

Let $h_{Q}(\xi)=\left(f(\xi)-f_{Q}\right) \chi_{S \backslash Q}(\xi)$. To complete the proof of Theorem 1.1, we need the following theorem.

Theorem 3.1. Let $f \in \operatorname{Lip}_{\alpha}(\xi)$, let $Q$ be a nonisotropic ball of radius $\delta$ and center at $\eta^{*}$, and let $\frac{1}{16} Q=\left\{\xi \in S:\left|1-\left\langle\xi, \eta^{*}\right\rangle\right|<\frac{\delta}{16}\right\}$. Suppose that there is an $\eta^{\prime} \in \frac{1}{16} Q$ so that $S_{\beta}\left(h_{Q}\right)\left(\eta^{\prime}\right)<\infty$. Then there exists a constant $C$, depending only on $n$ and $\beta$, such that $S_{\beta}\left(h_{Q}\right)(\eta)<\infty$ and $\left|S_{\beta}\left(h_{Q}\right)(\eta)-S_{\beta}\left(h_{Q}\right)\left(\eta^{\prime}\right)\right| \leq C \delta^{\alpha}\|f\|_{L i p_{\alpha}}$ for all $\eta \in \frac{1}{16} Q$.

Proof. Let $D_{\beta}^{-}(\eta)=D_{\beta}(\eta) \cap\left\{\frac{\beta r(z)}{2} \leq \frac{\delta}{16}\right\}$ and $D_{\beta}^{+}(\eta)=D_{\beta}(\eta) \cap\left\{\frac{\beta r(z)}{2}>\frac{\delta}{16}\right\}$.

We have

$$
\begin{aligned}
S_{\beta}\left(h_{Q}\right)(\eta)= & \left\{\int_{D_{\beta}(\eta)}\left|\widetilde{\nabla} \int_{S} P(z, \xi) h_{Q}(\xi) d \sigma(\xi)\right|^{2} r(z)^{-(n+1)} d \nu(z)\right\}^{\frac{1}{2}} \\
= & \left\{\int_{D_{\beta}^{-}(\eta)}\left|\widetilde{\nabla} \int_{S} P(z, \xi) h_{Q}(\xi) d \sigma(\xi)\right|^{2} r(z)^{-(n+1)} d \nu(z)\right\}^{\frac{1}{2}} \\
& \quad+\left\{\int_{D_{\beta}^{+}(\eta)}\left|\widetilde{\nabla} \int_{S} P(z, \xi) h_{Q}(\xi) d \sigma(\xi)\right|^{2} r(z)^{-(n+1)} d \nu(z)\right\}^{\frac{1}{2}} \\
= & \left\{\int_{D_{\beta}^{-}(\eta)}\left(\int_{S}|\widetilde{\nabla} P(z, \xi)|\left|h_{Q}(\xi)\right| d \sigma(\xi)\right)^{2} r(z)^{-(n+1)} d \nu(z)\right\}^{\frac{1}{2}} \\
& \quad+\left\{\int_{D_{\beta}^{+}(\eta)}\left(\int_{S}|\widetilde{\nabla} P(z, \xi)|\left|h_{Q}(\xi)\right| d \sigma(\xi)\right)^{2} r(z)^{-(n+1)} d \nu(z)\right\}^{\frac{1}{2}} \\
:= & I_{1}+I_{2} .
\end{aligned}
$$


By Lemma 2.1, we have

$$
\begin{aligned}
I_{1} \leq & n\left\{\int _ { D _ { \beta } ^ { - } ( \eta ) } \left(\int_{S \backslash Q}\left(\frac{\left(1-|z|^{2}\right)^{n}}{|1-\langle z, \xi\rangle|^{2 n}}+\frac{\left(1-|z|^{2}\right)^{n+1}}{|1-\langle z, \xi\rangle|^{2 n+1}}+\frac{\left(1-|z|^{2}\right)^{n+\frac{1}{2}}}{|1-\langle z, \xi\rangle|^{2 n+\frac{1}{2}}}\right)\right.\right. \\
& \left.\left.\times\left|f(\xi)-f_{Q}\right| d \sigma(\xi)\right)^{2} r(z)^{-(n+1)} d \nu(z)\right\}^{\frac{1}{2}} .
\end{aligned}
$$

If $z \in D_{\beta}^{-}(\eta)$, by Lemma 2.3 , we get

$$
\begin{gathered}
\left(\int_{S \backslash Q} \frac{\left(1-|z|^{2}\right)^{n}}{|1-\langle z, \xi\rangle|^{2 n}}\left|f(\xi)-f_{Q}\right| d \sigma(\xi)\right)^{2} \leq C(r(z))^{2 n} \delta^{-2(n-\alpha)}\|f\|_{L i p_{\alpha}}^{2}, \\
\left(\int_{S \backslash Q} \frac{\left(1-|z|^{2}\right)^{n+1}}{|1-\langle z, \xi\rangle|^{2 n+1}}\left|f(\xi)-f_{Q}\right| d \sigma(\xi)\right)^{2} \leq C(r(z))^{2 n+2} \delta^{-2(n+1-\alpha)}\|f\|_{L i p_{\alpha}}^{2}, \\
\left(\int_{S \backslash Q} \frac{\left(1-|z|^{2}\right)^{n+\frac{1}{2}}}{|1-\langle z, \xi\rangle|^{2 n+\frac{1}{2}}}\left|f(\xi)-f_{Q}\right| d \sigma(\xi)\right)^{2} \leq C(r(z))^{2 n+1} \delta^{-2\left(n+\frac{1}{2}-\alpha\right)}\|f\|_{L i p_{\alpha}}^{2} .
\end{gathered}
$$

It follows that

$$
\begin{aligned}
I_{1} \leq & C\left\{\int _ { D _ { \beta } ^ { - } ( \eta ) } \left[(r(z))^{2 n} \delta^{-2(n-\alpha)}+(r(z))^{2 n+2} \delta^{-2(n+1-\alpha)}\right.\right. \\
& \left.\left.+(r(z))^{2 n+1} \delta^{-2\left(n+\frac{1}{2}-\alpha\right)}\right] r(z)^{-(n+1)}\|f\|_{L i p_{\alpha}}^{2} d \nu(z)\right\}^{\frac{1}{2}} \\
= & C\left(\delta^{-2(n-\alpha)} \int_{D_{\beta}^{-}(\eta)}(r(z))^{n-1} d \nu(z)+\delta^{-2(n+1-\alpha)} \int_{D_{\beta}^{-}(\eta)}(r(z))^{n+1} d \nu(z)\right. \\
& \left.+\delta^{-2\left(n+\frac{1}{2}-\alpha\right)} \int_{D_{\beta}^{-}(\eta)}(r(z))^{n} d \nu(z)\right)^{\frac{1}{2}}\|f\|_{L i p_{\alpha}} \\
\leq & C\left(\delta^{-2(n+1-2 \alpha)} \int_{D_{\beta}^{-}(\eta)} d \nu(z)\right)^{\frac{1}{2}}\|f\|_{L i p_{\alpha}} .
\end{aligned}
$$

Arguing as in [2, P116], we get

$$
\int_{D_{\beta}^{-}(\eta)} d \nu(z) \leq C \delta^{n+1} .
$$

Hence

$$
I_{1} \leq C \delta^{\alpha}\|f\|_{L i p_{\alpha}} .
$$

Next, we estimate $I_{2}$ :

$$
\begin{aligned}
I_{2} & =\left\{\int_{D_{\beta}^{+}(\eta)}\left(\int_{S}|\widetilde{\nabla} P(z, \xi)|\left|h_{Q}(\xi)\right| d \sigma(\xi)\right)^{2} r(z)^{-(n+1)} d \nu(z)\right\}^{\frac{1}{2}} \\
& \leq\left\{\int_{D_{\beta}^{+}(\eta) \cap D_{\beta}\left(\eta^{\prime}\right)}\left(\int_{S}|\widetilde{\nabla} P(z, \xi)|\left|h_{Q}(\xi)\right| d \sigma(\xi)\right)^{2} r(z)^{-(n+1)} d \nu(z)\right\}^{\frac{1}{2}} \\
& +\left\{\int_{D_{\beta}^{+}(\eta) \backslash D_{\beta}\left(\eta^{\prime}\right)}\left(\int_{S}|\widetilde{\nabla} P(z, \xi)|\left|h_{Q}(\xi)\right| d \sigma(\xi)\right)^{2} r(z)^{-(n+1)} d \nu(z)\right\}^{\frac{1}{2}} .
\end{aligned}
$$


For the first term above, we have

$$
\begin{aligned}
& \left\{\int_{D_{\beta}^{+}(\eta) \cap D_{\beta}\left(\eta^{\prime}\right)}\left(\int_{S}|\widetilde{\nabla} P(z, \xi)|\left|h_{Q}(\xi)\right| d \sigma(\xi)\right)^{2} r(z)^{-(n+1)} d \nu(z)\right\}^{\frac{1}{2}} \\
& \leq\left\{\int_{D_{\beta}\left(\eta^{\prime}\right)}\left(\int_{S}|\widetilde{\nabla} P(z, \xi)|\left|h_{Q}(\xi)\right| d \sigma(\xi)\right)^{2} r(z)^{-(n+1)} d \nu(z)\right\}^{\frac{1}{2}} \\
& =S_{\beta}\left(h_{Q}\right)\left(\eta^{\prime}\right) .
\end{aligned}
$$

For the second term above, we have

$$
\begin{aligned}
& \left\{\int_{D_{\beta}^{+}(\eta) \backslash D_{\beta}\left(\eta^{\prime}\right)}\left(\int_{S}|\widetilde{\nabla} P(z, \xi)|\left|h_{Q}(\xi)\right| d \sigma(\xi)\right)^{2} r(z)^{-(n+1)} d \nu(z)\right\}^{\frac{1}{2}} \\
& \leq n\left\{\int _ { D _ { \beta } ^ { + } ( \eta ) \backslash D _ { \beta } ( \eta ^ { \prime } ) } \left(\int_{S \backslash Q}\left(\frac{\left(1-|z|^{2}\right)^{n}}{|1-\langle z, \xi\rangle|^{2 n}}+\frac{\left(1-|z|^{2}\right)^{n+1}}{|1-\langle z, \xi\rangle|^{2 n+1}}+\frac{\left(1-|z|^{2}\right)^{n+\frac{1}{2}}}{|1-\langle z, \xi\rangle|^{2 n+\frac{1}{2}}}\right)\right.\right. \\
& \left.\left.\quad \times\left|f(\xi)-f_{Q}\right| d \sigma(\xi)\right)^{2} r(z)^{-(n+1)} d \nu(z)\right\}^{\frac{1}{2}}
\end{aligned}
$$

Using Lemma 2.6, it follows that

$$
\begin{aligned}
& \left(\int_{S \backslash Q} \frac{\left(1-|z|^{2}\right)^{n}}{|1-\langle z, \xi\rangle|^{2 n}}\left|f(\xi)-f_{Q}\right| d \sigma(\xi)\right)^{2} \leq C(r(z))^{2 \alpha}\left(1+\left|\ln \left[\frac{r(z)}{\delta}\right]\right|\right)^{2}\|f\|_{L i p_{\alpha}}^{2}, \\
& \left(\int_{S \backslash Q} \frac{\left(1-|z|^{2}\right)^{n+1}}{|1-\langle z, \xi\rangle|^{2 n+1}}\left|f(\xi)-f_{Q}\right| d \sigma(\xi)\right)^{2} \leq C(r(z))^{2 \alpha}\left(1+\left|\ln \left[\frac{r(z)}{\delta}\right]\right|\right)^{2}\|f\|_{L_{i p_{\alpha}}}^{2}, \\
& \left(\int_{S \backslash Q} \frac{\left(1-|z|^{2}\right)^{n+\frac{1}{2}}}{|1-\langle z, \xi\rangle|^{2 n+\frac{1}{2}}}\left|f(\xi)-f_{Q}\right| d \sigma(\xi)\right)^{2} \leq C(r(z))^{2 \alpha}\left(1+\left|\ln \left[\frac{r(z)}{\delta}\right]\right|\right)^{2}\|f\|_{L i p_{\alpha}}^{2} .
\end{aligned}
$$

Hence

$$
\begin{aligned}
& \left\{\int_{D_{\beta}^{+}(\eta) \backslash D_{\beta}\left(\eta^{\prime}\right)}\left(\int_{S}|\widetilde{\nabla} P(z, \xi)|\left|h_{Q}(\xi)\right| d \sigma(\xi)\right)^{2} r(z)^{-(n+1)} d \nu(z)\right\}^{\frac{1}{2}} \\
& \leq C\left\{\int_{D_{\beta}^{+}(\eta) \backslash D_{\beta}\left(\eta^{\prime}\right)}\left(1+\left|\ln \left[\frac{r(z)}{\delta}\right]\right|\right)^{2} r(z)^{-(n+1-2 \alpha)}\|f\|_{L i p_{\alpha}}^{2} d \nu(z)\right\}^{\frac{1}{2}} .
\end{aligned}
$$

Notice that $\left|D_{\beta}^{+}(\eta) \backslash D_{\beta}\left(\eta^{\prime}\right) \cap\left\{r(z)=c^{\prime}\right\}\right| \leq C \delta^{n}$ for all $0<c^{\prime}<1$ (see [5, P128]) and $\frac{\beta r(z)}{2}>\frac{1}{16} \delta$. So we have $\left|D_{\beta}^{+}(\eta) \backslash D_{\beta}\left(\eta^{\prime}\right) \cap\left\{r(z)=c^{\prime}\right\}\right| \leq C \delta(r(z))^{n-1}$. Thus it follows that

$$
\begin{aligned}
& \left\{\int_{D_{\beta}^{+}(\eta) \backslash D_{\beta}\left(\eta^{\prime}\right)}\left(1+\left|\ln \left[\frac{r(z)}{\delta}\right]\right|\right)^{2} r(z)^{-(n+1-2 \alpha)}\|f\|_{L i p_{\alpha}}^{2} d \nu(z)\right\}^{\frac{1}{2}} \\
& \leq C \int_{\frac{\delta}{\delta \beta}}^{1}\left(1+\left|\ln \left[\frac{r(z)}{\delta}\right]\right|\right)^{2} \delta r(z)^{2 \alpha-2} d r(z)\|f\|_{L i p_{\alpha}} \\
& \leq C \delta^{\alpha} \int_{\frac{\delta}{8 \beta}}^{+\infty}(1+|\ln t|) t^{-2} d t\|f\|_{L i p_{\alpha}} \\
& \leq C \delta^{\alpha}\|f\|_{L i p_{\alpha}} .
\end{aligned}
$$

Using (3.3)-(3.5), we have

$$
I_{2} \leq S_{\beta}\left(h_{Q}\right)\left(\eta^{\prime}\right)+C \delta^{\alpha}\|f\|_{L i p_{\alpha}} .
$$


Combining (3.1) with (3.2), (3.6), it follows that

$$
S_{\beta}\left(h_{Q}\right)(\eta) \leq S_{\beta}\left(h_{Q}\right)\left(\eta^{\prime}\right)+C \delta^{\alpha}\|f\|_{L i p_{\alpha}} .
$$

Thus $S_{\beta}\left(h_{Q}\right)(\eta)$ is finite. Reversing the roles of $\eta$ and $\eta^{\prime}$, we obtain

$$
\left|S_{\beta}\left(h_{Q}\right)(\eta)-S_{\beta}\left(h_{Q}\right)\left(\eta^{\prime}\right)\right| \leq C \delta^{\alpha}\|f\|_{L i p_{\alpha}} .
$$

This completes the proof of Theorem 3.1.

The proof of Theorem 1.1. Write $f$ as

$$
\begin{aligned}
f(\xi) & =f_{Q}+\left(f(\xi)-f_{Q}\right) \chi_{Q}(\xi)+\left(f(\xi)-f_{Q}\right) \chi_{S \backslash Q}(\xi) \\
& =f_{Q}(\xi)+g_{Q}(\xi)+h_{Q}(\xi) .
\end{aligned}
$$

Since $f_{Q}$ is a constant, $S_{\beta}\left(f_{Q}\right)=0$. Thus $S_{\beta}\left(f_{Q}\right)$ is in $\operatorname{Lip} p_{\alpha}$ with the $\operatorname{Lip}_{\alpha}$ norm equal to 0 . Therefore

$$
\begin{aligned}
S_{\beta}(f) & \leq S_{\beta}\left(g_{Q}\right)+S_{\beta}\left(h_{Q}\right), \\
S_{\beta}\left(h_{Q}\right) & \leq S_{\beta}\left(g_{Q}\right)+S_{\beta}(f) .
\end{aligned}
$$

By the boundedness of $S_{\beta}(f)$ on $L^{p}(1<p<\infty)$ and the Cauchy-Schwarz inequality, we have

$$
\begin{aligned}
\int_{Q}\left|S_{\beta}\left(g_{Q}\right)\right| d \sigma(\xi) & \leq|Q|^{\frac{1}{2}}\left(\int_{Q}\left|S_{\beta}\left(g_{Q}\right)\right|^{2} d \sigma(\xi)\right)^{\frac{1}{2}} \\
& \leq C|Q|^{1+\frac{\alpha}{n}}\|f\|_{L_{i p_{\alpha}} .}
\end{aligned}
$$

It also follows from (3.9) that $S_{\beta}\left(g_{Q}\right)$ is finite almost everywhere. Therefore $S_{\beta}\left(h_{Q}\right)<+\infty$ at almost every point such that $S_{\beta}(f)<+\infty$.

Now, we prove $\left\|S_{\beta}(f)\right\|_{L i p_{\alpha}} \leq C\|f\|_{L i p_{\alpha}}$.

To show the boundedness of $S_{\beta}$ on $\operatorname{Lip}_{\alpha}$, by Lemma 2.2 and the triangle inequality, it suffices to show that for every $f \in \operatorname{Lip}_{\alpha}(S)$, there is a constant $\lambda=\lambda(Q, f)$ such that

$$
\frac{1}{|Q|^{1+\frac{\alpha}{n}}} \int_{Q}\left|S_{\beta} f(\xi)-\lambda\right| d \sigma(\xi) \leq C\|f\|_{L i p_{\alpha}} .
$$

Let $Q^{\prime} \subset S$ be any nonisotrophic ball, and $Q=16 Q^{\prime}$ (that is $Q^{\prime}=\frac{1}{16} Q$ ). Since $S_{\beta}\left(h_{Q}\right)(\eta)<+\infty$ at almost every point, we choose a point $\eta^{\prime} \in \frac{1}{16} Q$ so that $S_{\beta}\left(h_{Q}\right)\left(\eta^{\prime}\right)$ is finite. Then, by (3.9) and Theorem 3.1,

$$
\begin{aligned}
& \frac{1}{\left|Q^{\prime}\right|^{1+\frac{\alpha}{n}}} \int_{Q^{\prime}}\left|S_{\beta} f(\xi)-S_{\beta}\left(h_{Q}\right)\left(\eta^{\prime}\right)\right| d \sigma(\xi) \\
& =\frac{1}{\left|Q^{\prime}\right|^{1+\frac{\alpha}{n}}} \int_{Q^{\prime}}\left|S_{\beta}\left(g_{Q}+h_{Q}\right)(\xi)-S_{\beta}\left(h_{Q}\right)(\xi)+S_{\beta}\left(h_{Q}\right)(\xi)-S_{\beta}\left(h_{Q}\right)\left(\eta^{\prime}\right)\right| d \sigma(\xi) \\
& =\frac{1}{\left|Q^{\prime}\right|^{1+\frac{\alpha}{n}}} \int_{Q^{\prime}}\left|S_{\beta}\left(g_{Q}\right)(\xi)\right| d \sigma(\xi)+\frac{1}{\left|Q^{\prime}\right|^{1+\frac{\alpha}{n}}} \int_{Q^{\prime}}\left|S_{\beta}\left(h_{Q}\right)(\xi)-S_{\beta}\left(h_{Q}\right)\left(\eta^{\prime}\right)\right| d \sigma(\xi) \\
& \leq C\|f\|_{L i p_{\alpha}} .
\end{aligned}
$$

Since $Q^{\prime}$ is arbitrary, the proof is completed.

Remark 3.2. The referee asked whether the following proposition is true or not:

$$
S_{\alpha} f \in \operatorname{Lip}_{\alpha}(S) \Rightarrow f \in \operatorname{Lip}_{\alpha}(S) ? ?
$$

The authors cannot give a positive proof right now. 


\section{ACKNOWLEDGEMENT}

The authors express their gratitude to the referee for very valuable comments.

\section{REFERENCES}

1. W. Rudin, Function Theory in the unit ball of $C^{n}$, Springer-Verlag, New York, 1980. MR0601594 (82i:32002)

2. S. Y. Chang, A generalized area integral estimate and applications, Studia Math. 69 (1980), 109-120. MR0604343 (82d:42014)

3. P. Ahern and J. Bruna, Maximal and area integral characterization of Hardy-Sobolev spaces in the unit ball of $C^{n}$, Rev. Mat. Iber. 4 (1988), 123-153. MR1009122 (90h:32011)

4. S. G. Krantz, Geometric Lipschitz spaces and applications to complex function theory and nilpotent groups, J. Func. Anal. 34 (1979) 456-471. MR0556266 (81j:32020)

5. H. Kang and H. Koo, Two weight inequalities for the derivatives of holomorphic functions and Carleson measures on the unit ball, Nagoya Math. J. 58 (2000), 107-131. MR1766570 (2002b:32008)

6. E. M. Stein, Singular Integrals and Differentiability properties of Functions, Princeton Univ. Press, Princeton, 1970. MR0290095 (44 \#7280)

7. S.G. Krantz and S. Y. Li, Area integral characterizations of functions in Hardy space on domains in $C^{n}$, Complex Variables Theory Appl. 32 (1997), 373-399. MR1459599 (98m:32003)

8. A. Bonami, J. Bruna and S. Grellier, On Hardy, BMO and Lipschitz spaces of invariantly harmonic functions in the unit ball, Proc. Lond. Math. Soc. 77 (1998), 665-696. MR1643425 (99g:31008)

Department of Mathematics, Fujian Normal University, Fuzhou, 350007, People's Republic of China

Current address: Department of Mathematics, Zhejiang University, Hangzhou, 310028, People's Republic of China

E-mail address: gaojinshou@yahoo.com.cn

Department of Mathematics, Zhejiang University, Hangzhou, 310028, People's RePUBLIC OF CHINA

E-mail address: mjhy@zju.edu.cn 